\title{
THE TREATMENT OF ACUTE LOBAR PNEUMONIA.
}

\author{
$B y$ G. J. LANGLEY, M.D., F.R.C.P. (Lond.).
}

(Reader in General Therapeutics, Victoria University; Hon. Physician, Ancoats Hospital, Salford, Royal Hospital, Manchester; and Visiting Physician, Hope Hospital, Salford.)

\section{INTRODUCTORY.}

The incidence of lobar pneumonia in our great cities is sufficiently large to make the disease one of great importance, for its mortality is still also very high, varying though it does in every epidemic. But the day is now happily past when the medical attendant upon these distressing and urgent cases was obliged to feel that any part which he could play in their recovery was a very minor one. There is now almost universal concensus of opinion among those who have made any extended trial of the modern concentrated antipneumococcal serum, that this offers a means of curtailing the attack and probably of saving life.

For such advances as have been made during recent years we chiefly have to thank our American cousins, as we have also to turn to their careful and voluminous records with their large numbers, for evidence of the value of serum treatment.

The treatment of this disease may for descriptive purposes be divided under two heads (I) general and (2) specific, and in each case it is imperative that all our resources be used at the earliest possible moment. Such must involve early diagnosis and although this is often easy in the presence of a sudden rigor, yet it is a matter of experience that the vomiting at onset, which is common even in adults, proves to be misleading to many.

\section{GENERAL MEASURES.}

The sudden and severe malaise will call for complete rest in bed, and the disturbed respiration is often relieved by a high bedrest and sufficient pillows to form a "chair" in which the patient can relax fully without slipping.

Pleuritic Pain. The severe pain in the chest is usually treated by poultices or antiphlogistine, care must, however, be taken that the weight of the application, or the tightness of the retaining bandage, shall not impede the disturbed respiration. The utility of such applications usually disappears by the end of the third day, either because the pain is reduced or the patient is too toxic to be benefited.

Cough. Cough is a variable factor, in some cases producing great accession of pain and discomfort. It is frequently produced by an associated bronchitis, more particularly in that group of cases which arise in patients who have been liable to recurrent attacks of chest trouble. In such there may be considerable fluid sputum, expulsion of which appears to relieve the condition to some extent. And for these the ordinary expectorants appear to be useful. For that other and larger group, however, in which spasms of severe cough result in only very small, viscid masses of adhesive mucus, the expectorants appear useless and sedatives seem to be the correct treatment. 
Sleeplessness. So much has been written, and still more said, upon the vexed question of sleep in pneumonia, that a definite statement of opinion is almost all that can be attempted here. The rules laid down in a "Service" which treats 200 cases of pneumonia per year may thus be sufficient.

It is considered that a restless and sleepless night calls for some drug on the following night to ensure sleep. For this purpose three alternatives are offered, (I) medinal gr. Io, (2) chloral and potass. bromid. aa. gr. 20, (3) paraldehyde dr. I $\frac{1}{2}$ dissolved in water ozs. $3 \frac{1}{2}$ and given per rectum after an enema.

Experience in using these three has resulted in the view that they all frequently fail, but that the last is the most efficient. In the common event of their failure to act there is a definite call on the succeeding night for a quarter of a grain of morphia administered hypodermically. This routine is used quite irrespective of the day of disease, the only accepted contraindication to morphia being a profuse expectoration with widespread bronchitis. It is considered that the restless distress of a bad night will do far more harm than is ever likely to result from the morphia itself.

Heart Failure. If much has been written with regard to suitable drugs for sleep, still more has been produced respecting drugs designed to " support the heart". It is held that the only relief attainable to the circulation is to be found in the curtailment or termination of the toxic process itself. In the routine treatment of a relatively large number of cases per year, a definite procedure has been attempted. It is believed that digitalis is useless except in those cases which develop auricular fibrillation and even here it is a matter of experience that this abnormal rhythm may only persist for a few hours at a time in many instances. When fibrillation has been observed, a standard tincture of digitalis is given in drachm doses every two hours until either the rhythm has returned to normal, or the heart speed has been reduced to about Ioo per minute. In any case, after such an occurrence the patient is kept partly under digitalis control until the crisis has occurred, the maintenance dose being taken as $\mathrm{dr}$. $\frac{1}{2}$ of the tincture daily.

Strychnine is also used, given hypodermically in doses of I/30 gr. four hourly, when the toxic condition has become very severe, aiming rather at the effect upon the respiratory centre, than hoping for any effect upon the heart itself.

Dyspnoea and Cyanosis. The administration of oxygen offers yet another problem and this is used in the pneumonia "Service" for two purposes (I) to relieve cyanosis when severe and (2) to relieve the distress of rapid respiration. It is always administered through a nasal catheter to which patients rarely object although its position is often disturbed in delirium. The oxygen is passed through water, partly to avoid any drying effect of the gas but chiefly to enable the rate of flow to be easily checked and maintained at a constant rate. The entry tube is fixed one inch below the water level and the bubbles regulated to roughly one per second. This is maintained constantly, day and night when required, and the actual volume of oxygen used is small. When the high rate of respiration is the factor calling for oxygen administration a mixture containing $5 \% \mathrm{CO}_{2}$ is employed. The use of the oxygen tent or chamber has not been found practicable in a "Service" which treats so many cases, and the cost of which is still very high.

So far it has proved almost impossible to gauge the effect of this treatment upon patients and no attempt has been made in this "Service" to estimate the degree of change in oxygen saturation produced by the method of administration adopted. 
The use of the steam tent and the bronchitis kettle has been entirely given up, the patients instead being nursed in a well ventilated ward. It is our experience that patients in great respiratory distress will welcome being placed near a widely open window, even on cold days, always provided they are well covered with blankets and supplied with hot water bottles. The temperature of the wards in which these patients are nursed is usually below that of the other wards in the Hospital and it is very rare for any patient to complain. At the same time it is held that free ventilation is an important factor in guarding against infection to other patients and to those in attendance in the ward. It is further held that the same arguments apply when treating patients in their own homes or in a Nursing Home. Instances of infection occurring in the wards do still present themselves from time to time, but fortunately so far, no ward epidemic has occurred, in spite of the fact that two or three members of the same family have occasionally been admitted at the same time.

Diet. Diet during the attack is of the usual fluid or semi-fluid type, the only special feature is that fluid intake and output is recorded daily and that glucose is used very plentifully in all drinks. Glycosuria as the result of this only occurs with great rarity. After the crisis it is usual to order ol. morrhuæ in the hope that its vitamin content may help forward resolution.

Stimulants are not used as a routine although brandy, six ounces daily, is commonly ordered for those who are most urgently ill. A mixture containing sp. aetheris and sp. ammon. aromat. aa. dr. $\frac{1}{2}$ is similarly used, occasionally with apparently satisfactory results.

\section{SPECIFIC MEASURES.}

Necessity for typing Causative Organism. Turning now to the specific treatment of the disease, it is obviously imperative that arrangements should exist for the immediate typing of all pneumonia cases. Immediately after admission a sample of sputum, which must be quite fresh, is sent to the laboratory for typing. Four years of this work has made it possible for eighty per cent. of the cases to be typed correctly direct from the sputum. The method used is that described by Armstrong, making use of the capsular swelling which is known to take place in the pneumococcus in the presence of its homologous antibody. By this means the type of the causative organism can be known within a few hours of the patient's admission to Hospital.

Class of Case treated. Serum is only given in this "Pneumonia Service" to patients admitted to hospital before the end of the third day of the disease and who are suffering from Type I or Type 2 pneumonia. The reasons for this rule are that the first two types are the only ones for which serum is at present available; that the evidence of benefit after the third day is not very marked, as revealed in the large American figures; and that the cost of adequate serum is still very high.

Serum used. The concentrated serum, monovalent and of the Felton type (concentrated globulin), is that which is employed. It is always given intravenously and only causes reactions on rare occasions. It is wise to enquire of the patient or his friends as to the occurrence of asthma, or of the giving of any serum for any purpose before. A hypodermic syringe containing one cc. of one in a thousand adrenalin solution should always be in readiness. The small volume of the concentrated serum, and the fact that it contains only the globulin fraction renders serum reactions improbable. 
Dose of Serum. The dose of the serum to be administered has been much discussed and there are great differences of opinion as to what the initial dose should be. Reasons have been advanced for giving increasing doses according to the day of the disease upon which the treatment is started, and for giving twice the dose of Type 2 serum which would have been given for Type I cases. Further, it has been suggested that a much larger dose should be given to those cases which yield a positive blood culture than those without bacteræmia.

The routine dose employed in the "Pneumonia Service" is 40,000 units given intravenously and it is expected that further subsequent doses will be required.

It has been found by others as well as in this "Service", that just before or immediately after the crisis, a substance can be demonstrated in the serum of the patient which will cause agglutination and capsular swelling in a recent living virulent culture of the homologous organism. In view of this fact it is the routine practice in this "Service" to test the patient's blood for antibody four hours after serum administration. If no reaction can be obtained a further dose of 40,000 units of serum is administered and the antibody test is again applied, serum and test being repeated every four or six hours until persistent antibody can be demonstrated in the patient's serum.

It has been our experience that fall in temperature and clinical improvement as the result of serum administration does not necessarily indicate adequate dosage; for the whole condition may have relapsed within the next ensuing twelve hours. The persistent presence of antibody in the patient's serum after treatment has, however, proved to be a reliable guide in our hands, although several observers are known not to hold this view. It is felt that the further investigation of this important question lies with the pathologist rather than with the clinician and the literature is already voluminous. It is believed, however, that there is a definite reaction between antigen and antibody which results in the using-up of available antibody by antigen and further that crisis with recovery is not possible until available antibody exists in the patient's serum beyond that which is fixed by the antigen.

Bacteræmia. There is very good reason for regarding those cases as most grave which show a positive blood culture, and it is the experience of this "Service" that the result of blood culture is of the utmost value in determining prognosis. There seems, however, to be a real difference between cases which show a bacteræmia before the end of the third day as some of these seem to run a relatively mild course, whereas those which show organisms in the blood after the sixth day, show a very high mortality indeed. It appears to workers in the "Service" that the daily results of blood culture are among the most valuable of the data available to the clinician in treating this serious and difficult disease.

There is also evidence that the late bacteræmic cases i.e. those showing a positive blood culture from the sixth day onwards, frequently already have purulent pleurisy or pericarditis; whether their chances of recovery would be increased by daily aspiration of these purulent accumulations has not yet been determined, but it would be perhaps a justifiable attempt in spite of the grave condition of the patients.

It is felt by many that an attempt should be made to treat by means of serum, the patient who comes under observation for the first time on the fifth or sixth day of the disease and who is obviously gravely ill, in the hope that it may help him over 
the final stage of the illness. The evidence, in bulk, is against any such hope, but it must be obvious that its trial in any individual case carries no contraindication, although the required dose of the serum is sure to be large and therefore costly.

\section{IMPORTANCE OF COMPLICATIONS.}

There is probably no condition more trying to the physician than the patient with a frank pneumonia who, after the tenth day, has still failed to show improvement or have a crisis. It is true that crisis has been observed in this "Service" so late as the sixteenth day, but it is also true that delay up to, and after the tenth, day suggests some complication which may not be easily discovered. A persistent bacteræmia will suggest that some focus other than the lung is in existence and a small proportion of cases do develop an ulcerative endocarditis which may not show itself in the form of a marked cardiac lesion until very late in the disease. Early empyema or a pneumococcal arthritis may also escape detection but fortunately pneumococcal meningitis, hidden though it may be by the delirium of the disease, is far from common. On the other hand, a pneumococcal lumbar slough, mistaken for a bedsore, has proved misleading on more than one occasion. In any case it would seem wise to regard a diagnosis of unresolved pneumonia with the gravest possible suspicion, and not to accept it readily from day to day as an adequate explanation of continued illness and pyrexia, unless every other possible explanation can be really excluded.

There is a small group of cases in which the crisis is not followed promptly by that degree of clinical recovery to which one is accustomed; delirium at night may persist for some days or the appetite fails to make any improvement. Most of these cases do ultimately recover fully, but a long time is liable to be occupied in the process.

Empyema. No review of the treatment of pneumonia can be complete without reference to empyema. As already pointed out, this may occur early in the disease, before the ninth day, and at this stage of the disease it is liable to be very fatal, since in our experience it is almost always associated with a persistent bacteræmia. The only possible treatment would appear to be repeated aspiration and even then the issue is usually unhappy.

Empyema, however, is as a rule discovered a few days after the crisis, the temperature most usually remaining normal; diagnosis is completed by means of the exploring syringe.

Provided the pus is localised by adhesions almost any measure for its removal will be successful, but when such adhesions are absent, or the extent of the abscess is very large, a wide thoracotomy is in our experience disastrous.

Closed drainage in such cases seems to be the only justifiable treatment and, where doubt exists as to the extent of the empyema cavity, this can sometimes be determined by aspirating Ioo cc. of pus, replacing it by air, and outlining the cavity by changing the position of the patient against the $\mathrm{X}$-ray screen. In this way it may be possible to determine the choice between open and closed drainage. The obvious solution of the problem by invariably making use of the closed drainage method is not so lightly to be undertaken, since the care and attention required to maintain closed drainage are not always forthcoming outside the special wards of a large hospital. 